\title{
Utilização do sensor sonda lambda como sensor de pressão negativa.
}

\author{
Valli, F. V. ; Rodrigues, J. P. ${ }^{1}$; Del Caro, R. L. C. ${ }^{1}$; Batisti, R. N. ${ }^{1}$; Prado, A. R. ${ }^{1}$ \\ 1 Instituto Federal do Espírito Santo, Serra, ES, Brasil.
}

\begin{abstract}
Resumo
Os sensores estão massivamente incorporados ao cotidiano de diversos segmentos. O sensor de pressão é bastante utilizado tanto em ambientes domésticos como em vários segmentos da indústria. Porém, quando se trata de precisão, o alto custo se torna bastante evidente entre eles. Neste trabalho, busca-se comprovar que o sensor de oxigênio, mais conhecido como sonda lambda, que é um sensor relativamente barato, utilizados em carros, pode ser adaptado para medir pressão em ambientes confinados. Essa prova é baseada nos fundamentos teóricos já utilizados nesse sensor, que são a equação de Nernst e a lei de pressões parciais de Dalton, onde permite obter uma relação entre tensão de saída do sensor e pressão do recipiente medido. Será criado um setup para a realização dos experimentos, e por questões de segurança e limitação do escopo do projeto, inicialmente, serão usados somente pressão negativa. Para a aquisição dos dados serão usados a plataforma de prototipagem Arduino Mega e o software Labview da National Instruments, juntamente com filtros que serão projetados. Posteriormente, para tratamento dos dados será utilizado o programa Microsoft Excel, onde por final será obtido uma equação que forneça a tensão de saída em função da pressão medida, para comprovar as teorias.
\end{abstract}

Palavras chaves: Sensor de pressão. Sonda Lambda. Equação de Nernst. Sistema de aquisição de dados.

\begin{abstract}
The sensors are massively incorporated into everyday life of several segments. Pressure sensor is widely used in both domestic and multi-industry environments. However, when it comes to accuracy, the high cost becomes quite evident among them. In this work, we seek to prove that the probe lambda (oxygen sensor) which is a relatively inexpensive sensor, used in cars, can be adapted to measure pressure in confined environments. This proof is based on the theoretical foundations already used in this sensor, which are the Nernst equation and the Dalton partial pressure law, where it allows to obtain a relation between sensor output voltage and measured pressure. A setup will be created to perform the experiments, and for safety reasons and project scope limitation purposes, only negative pressure (vacuum) will be used. Arduino Mega prototype platform and Labview software by National Instruments will be used to acquire the data, along with electronic filters that will be designed. Then, for data treatment, Microsoft Excel program will be used, whereby an equation will be obtained that provides the output voltage as a function of the measured pressure, to prove the theories.
\end{abstract}

Keywords: Pressure sensor. Lambda sensor. Nernst equation. Data acquisition system.

\section{Introdução}

Atualmente, os sensores estão presentes ao cotidiano de diversos segmentos. Sua importância deve-se prioritariamente aos inúmeros espaços onde o mesmo pode ser utilizado. A importância deste instrumento se dá devido ao grau de desenvolvimento da sociedade industrializada, no qual pode ser avaliado o uso que esta faz de instrumentos de medição definidos como dispositivos para detectar, medir, registrar ou controlar a variação de parâmetros em um processo. Nos laboratórios e fábricas em todo o mundo, a demanda para este tipo de equipamento é cada vez maior, para que a fabricação e a qualidade de um produto possam ser adequadamente monitoradas [1].

Portanto, o desenvolvimento de novos sensores e a aplicação adequada dos mesmos requer um profundo conhecimento dos fundamentos teóricos e dos princípios de funcionamento envolvidos. $\mathrm{Na}$ sociedade contemporânea, suas aplicações se diferenciam, e para 
tanto, há diversos sensores existentes na atualidade, como: sensor de temperatura, pressão, umidade, aceleração, direção, ângulo, fluxo, nível, deslocamento, presença, posição, força, torque, entre outros.

Dentre todos os sensores existentes no mercado, o sensor de pressão é um bastante utilizado, até mesmo no ambiente doméstico (botija de gás), assim como na indústria de petroquímicos, segmentos automotivos, eletrônicos de consumo, utilitários e segmento industrial [2]. Esse sensor de pressão, o qual pode ser usado em várias aplicações será objeto desse estudo para embasamento do projeto a ser desenvolvido (executado).

Esse trabalho objetiva criar um sensor de pressão de ar através de um sensor sonda lambda, que é utilizado em sua essência como um sensor de oxigênio em carros para verificar se a combustão foi realizada com sucesso e para que esse valor de oxigênio contribua no controle de injeção eletrônica. Dessa forma, utilizaremos os fundamentos teóricos usados no medidor sonda lambda, como a equação de Nernst e lei de pressões parciais de Dalton para achar uma relação direta entre sua tensão de saída e a pressão de ar do ambiente medido, de forma a se obter uma possível equação para a resposta do sensor.

\section{Materiais e Métodos}

Para verificar a possiblidade de se criar um sensor de pressão a partir do sensor Sonda lambda foi analisado a equação que rege esse instrumento que é a equação de Nernst [3] e é dada por

$$
U s=\frac{R * T}{4 * F} * \ln \left(p O 2^{\mathrm{w}} / p O 2^{t}\right)
$$

onde

Us = Diferença de potencial $(\mathrm{V})$

$\mathrm{R}=$ Constante geral dos gases $(8,3143 \mathrm{~J} / \mathrm{gmol} \mathrm{K})$

$\mathrm{F}=$ Constante de Faraday $(96485,3 \mathrm{C} / \mathrm{gmol})$

$\mathrm{T}=$ Temperatura absoluta $(\mathrm{K})$

$\rho \mathrm{O} 2 "$ = Pressão parcial de oxigênio da carga de referência (atmosfera).

$\rho \mathrm{O} 2$ ' = Pressão parcial de oxigênio no gás do processo.

Nela é possível obter uma relação entre a tensão de saída do sensor (Us) e pressão parcial de oxigênio do recipiente a ser medido ( $\mathrm{pO} 2$ '). Deve-se considerar que o R, F, T e pO2" são constantes. Vale ressaltar que os testes realizados nesse trabalho foram feitos somente em pressão de ar, o que nos fornece que a composição de oxigênio no ar se mantêm sempre constante e igual a aproximadamente $21 \%$ [4].
Para concluir e complementar a teoria utilizada nesse projeto foi feito uso das leis parciais de Dalton [4], dada por

$$
P_{t}=\frac{P_{P_{x}}}{x_{x}}
$$

onde

$P_{t}=$ Pressão Total da mistura dos gases .

$P_{x}=$ Pressão parcial do gás $X$ da mistura.

$X_{x}=$ Fração Molar do gás $\mathrm{X}$ da mistura.

A partir da equação 2 podemos justificar inicialmente o motivo de termos considerado constante o pO2", pois já que pressão parcial de oxigênio da atmosfera, a fração molar de oxigênio é constante e em $21 \%$ e a pressão total da atmosfera também é constante, o que nos dá a pressão parcial de oxigênio da atmosfera (pO2") também constante. Seguindo o mesmo princípio descrito anteriormente e considerando que estamos medindo pressão de ar em um recipiente, ou seja, a composição desse ar também é sempre a mesma, tendo posse da tensão de saída do sensor podemos obter a partir da equação 1 a pressão parcial de oxigênio do recipiente pO2'. Aplicando pO2' a equação 2 e considerando que a fração molar de oxigênio sempre se mantém constante (21\%) por estarmos medindo pressão de ar, podemos obter por manipulação matemática o valor da pressão total do recipiente, o que é o objetivo desse trabalho.

Com base na teoria, foi criado um setup de teste com o uso dos seguintes materiais:

- $\quad$ Sensor sonda Lambda DPL608416 12V de 4 fios, como mostra a figura 1.

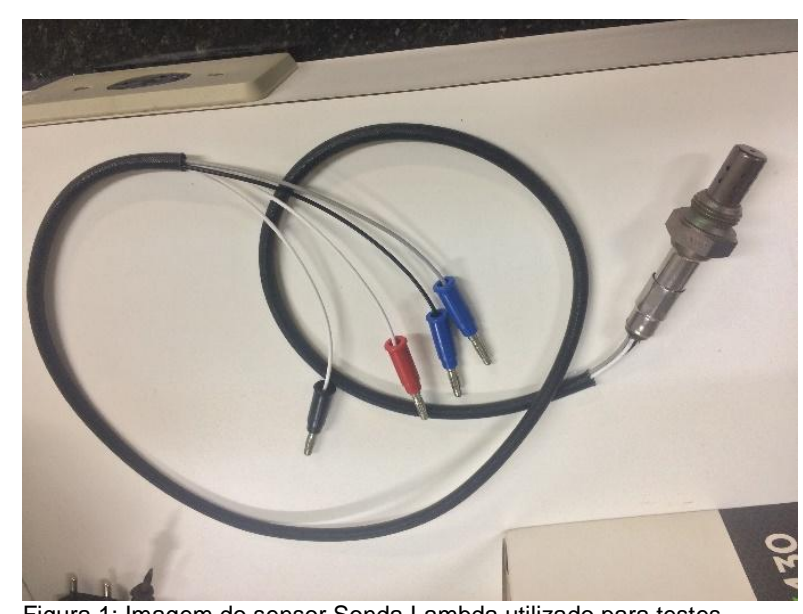

Figura 1: Imagem do sensor Sonda Lambda utilizado para testes.

- Bomba de vácuo da Prismatec com capacidade de fornecer uma pressão entre -15 psi até -30 psi. Foi utilizado somente pressão 
negativa por motivos de segurança do laboratório.

- Tanque com capacidade de 1,42 litros, em que será aplicado a pressão de teste.

- Fonte de Alimentação DC da Politerm para a alimentação do sensor Sonda lambda.

- Arduino Mega mais o circuito de condicionamento do sinal (filtro ativo passa baixa) para a aquisição dos dados do sensor.

- Computador onde estará o supervisório feito no software Labview da National Instruments e onde será feito o tratamento dos dados no Microsoft Excel.

Com o uso desses materiais foi obtido o seguinte setup, como mostra a figura 2.

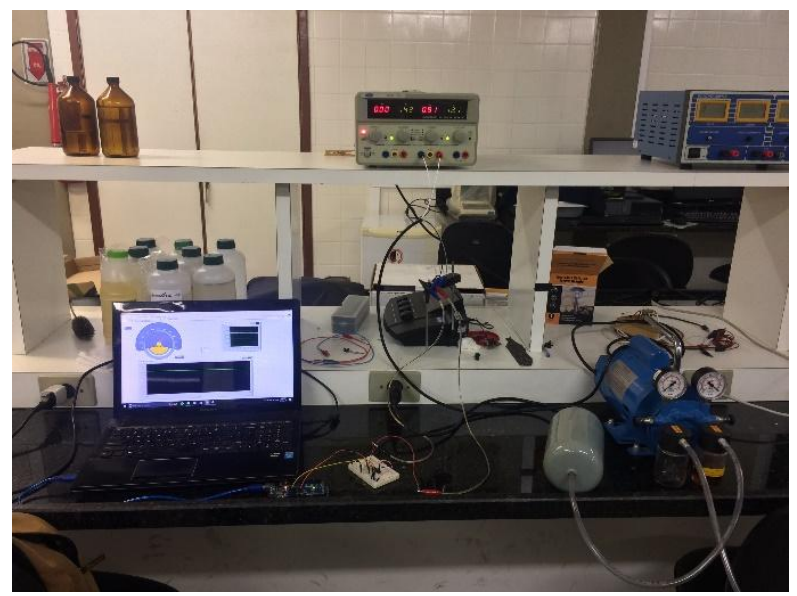

Figura 2: Imagem do sensor Sonda Lambda utilizado para testes.

Antes de se iniciar definitivamente os testes, foi criado um roteiro para a realização dos mesmos, de modo a se ter resultados confiáveis e que possam ser repetidos para se evitar qualquer alteração desses resultados devido a fatores externos. As repetibilidade de ensaios em laboratórios são cruciais para a comparação e conclusão dos mesmos. Para isso, foi seguido o seguinte roteiro enumerado abaixo. Lembrando que o tempo de amostragem utilizado foi de $10 \mathrm{~ms}$.

1.Colocar a bomba em $0 \mathrm{mmHg}$.

2.Ligar a bomba.

3.Capturar os dados por 20s, que nos dá 2000 amostras para um tempo de amostragem de 10ms (100 amostras por segundo).

4.Desligar a bomba.

5.Esperar 1 minuto.

6. Realizar os tópicos de 1 a 5 para os valores de pressão $-100 \mathrm{mmHg},-200 \mathrm{mmHg},-300 \mathrm{mmHg},-400$ $\mathrm{mmHg}$ e $-500 \mathrm{mmHg}$, respectivamente.

Foram realizados os tópicos acima três vezes, com um intervalo entre cada um de 10 minutos. O tempo de um minuto dado no tópico 5 foi em razão do aquecimento que a bomba sofre quando fica muito tempo ligada, então esse foi um meio para se evitá-lo.

\section{Resultados e Discussões}

Para cada uma das pressões, os dados foram exportados para o Microsoft Excel para uma melhor análise. Considerando que durante os 20 segundos de duração dos testes realizados, o sinal obtido estava estabilizado para uma única pressão, foi retirado a média das 2000 amostras de cada uma das pressões de cada um dos testes para que conseguirmos obter um valor que facilite o entendimento. Assim foram obtidas as seguintes tabelas 1, 2 e 3 .

Tabela 1: Tabela com a média das 2000 amostras de cada uma das pressões para o teste 1 .

\begin{tabular}{c|c}
\hline $\begin{array}{c}\text { Pressão aplicada } \\
\text { (mmHg) }\end{array}$ & Tensão de saída (Teste 1) \\
\hline 0 & 0.07451 \\
-100 & 0.07633 \\
-200 & 0.075176 \\
-300 & 0.073454 \\
-400 & 0.071543 \\
-500 & 0.069675 \\
\hline
\end{tabular}

Tabela 2: Tabela com a média das 2000 amostras de cada uma das pressões para o teste 2 .

\begin{tabular}{c|c}
\hline $\begin{array}{c}\text { Pressão aplicada } \\
\text { (mmHg) }\end{array}$ & Tensão de saída (Teste 2) \\
\hline 0 & 0.078938 \\
-100 & 0.078261 \\
-200 & 0.076017 \\
-300 & 0.074157 \\
-400 & 0.071627 \\
-500 & 0.067332 \\
\hline
\end{tabular}

Tabela 3: Tabela com a média das 2000 amostras de cada uma das pressões para o teste 3 .

\begin{tabular}{c|c}
\hline $\begin{array}{c}\text { Pressão aplicada } \\
\text { (mmHg) }\end{array}$ & Tensão de saída (Teste 3) \\
\hline 0 & 0.07934 \\
-100 & 0.079118 \\
-200 & 0.077551 \\
-300 & 0.074848 \\
-400 & 0.072641 \\
-500 & 0.069479 \\
\hline
\end{tabular}

Com as tabelas 1, 2 e 3 podemos comprovar o que já esperávamos sobre a teoria da equação de Nernst, onde mostra que o sensor sonda lambda possui uma relação diretamente proporcional entre a tensão de saída e a pressão medida. Mas vale ressaltar, que teoricamente, de acordo com a equação de Nernst, essa relação é inversamente proporcional. Entretanto, pelo fato de termos referenciado o sensor no cabo do sinal (cabo preto do sensor usado), essa relação se inverteu. Essa inversão foi feita por limitações do Arduino MEGA não conseguir ler sinais analógicos negativos, o que era exatamente o que estava acontecendo quando o sensor estava no ambiente de 
teste. Então para solucionar esse problema foi feito essa troca de referência.

De posse dos dados dos três testes, devemos agora descobrir se para uma mesma pressão a tensão de saída se comporta de uma mesma maneira nos três diferentes momentos, isso comprovaria a repetibilidade do sistema. Uma técnica estatística bastante usada é a chamada coeficiente de variação, onde é expressado a variabilidade (dispersão) dos dados em porcentagem [5]. O cálculo do coeficiente de variação é feito através da equação 3:

$$
C V=\frac{s}{\mathrm{~S}} * 100
$$

Em que,

$\mathrm{S}=$ é o desvio padrão amostral

$\bar{x}$ = é a média dos dados

$\mathrm{CV}=$ é o coeficiente de variação

Quanto menor for o coeficiente de variação, mais homogêneos serão os dados. Geralmente, se aplica a seguinte ordem para os valores de CV:

- $\quad \mathrm{CV}<=15 \%$ indica baixa dispersão;

- $\mathrm{CV}>15 \%$ e CV $<=30 \%$ indica média dispersão;

- $\quad \mathrm{CV}>=30 \%$ indicia alta dispersão.

Assim sendo, foi obtido uma tabela com o desvio padrão amostral (feito pelo Excel com a função STDEVA), a média, e por final o Coeficiente de Dispersão para todas as pressões e todos os três testes, como mostra a tabela 4.

Tabela 4: Tabela com desvio padrão amostral, tensão de saída média e coeficiente de variação dos três testes desenvolvidos.

\begin{tabular}{c|c|c|c}
\hline $\begin{array}{c}\text { Pressão } \\
\text { aplicada } \\
\text { (mmHg) }\end{array}$ & $\begin{array}{c}\text { Tensão de } \\
\text { saída } \\
\text { (MÉDIA) }\end{array}$ & $\begin{array}{c}\text { Desvio } \\
\text { padrão } \\
\text { amostral }\end{array}$ & $\mathbf{C V ( \% )}$ \\
\hline 0 & 0.077596 & 0.002680 & 3.454 \\
-100 & 0.077903 & 0.001428 & 1.833 \\
-200 & 0.076248 & 0.001204 & 1.579 \\
-300 & 0.074153 & 0.000697 & 0.940 \\
-400 & 0.071937 & 0.000611 & 0.850 \\
-500 & 0.068829 & 0.001300 & 1.889 \\
\hline
\end{tabular}

Portanto, a tabela 4 mostrou através da coluna CV(\%), basicamente, como os dados foram realmente homogêneos, pois todos os valores de CV se mantiveram muito abaixo do limite de $15 \%$. Isso comprova também, a repetibilidade do roteiro de testes desenvolvido e do sensor, pois foram capazes de fornecer três dados em três momentos diferentes com uma boa proximidade entre eles. Este resultado nos mostra, principalmente, como o sensor sonda lambda pode ser confiável trabalhando como um sensor de pressão nesse range utilizado.

Dessa forma, podemos agora utilizar a Tensão de saída (MÉDIA) da tabela 4 como referência para o resultado final, pois é nele que contém informações dos três experimentos, por ser uma média dos mesmos. $\mathrm{Na}$ figura 3 é possível ver o gráfico da Tensão de saída (MÉDIA).

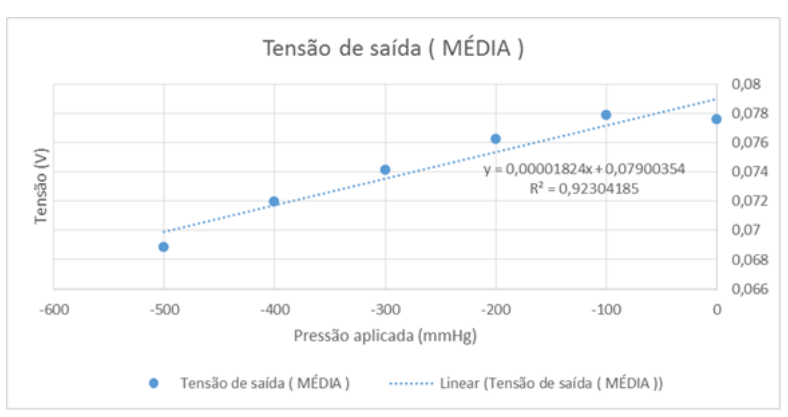

Figura 3: Gráfico da Tensão de saída média de todos os três experimentos. Junto se encontra uma regressão linear (reta com pontos poucos espaçados), a equação da reta e o coeficiente de Pearson que é usado para saber quão próximo essa reta está dos dados. Quanto mais próximo de 1 o R2 está, melhor é o modelo da reta em relação aos dados, o que faz a regressão linear da figura ser muito boa por obter um valor de 0,923.

Todos os gráficos nos mostraram dados bastantes satisfatórios e de acordo com o que esperávamos. Portanto como pode ver no gráfico da figura 3 , foi usado uma regressão linear que é uma equação que estima a linha pontilhada de forma a se obter uma fórmula que fornece a tensão de saída do sensor em y em função da pressão em $x$. Neste caso a equação 4 foi:

$$
y=0.00001824 * x+0.07900354
$$

Essa foi a equação que esse trabalho resultou do uso do sensor sonda lambda quando usado para medir pressões negativas nesse range de 0 a $-500 \mathrm{mmHg}$. Nela podemos aplicar as pressões utilizadas durante os testes e observarmos o comportamento de um possível tipo desse sensor, assim como o erro entre os valores obtidos pela equação e pelo sensor real, como é possível ver na tabela 5 .

Tabela 5: Possíveis tensões de saída em Volts utilizando a equação 3 obtida para as mesmas pressões usadas durante esse projeto comparadas a Tensão de Saída (Média) da tabela 4.

\begin{tabular}{c|c|c|c}
\hline $\begin{array}{c}\text { Pressão } \\
\text { aplicada } \\
\text { (mmHg) }\end{array}$ & $\begin{array}{c}\text { Tensão de } \\
\text { saída pela } \\
\text { equação 3 } \\
(\mathbf{V})\end{array}$ & $\begin{array}{c}\text { Tensão } \\
\text { de saída } \\
\text { real } \\
\text { (MÉDIA) }\end{array}$ & Erro (\%) \\
\hline 0 & 0.07900354 & 0.077596 & 1.81393371 \\
-100 & 0.07717954 & 0.077903 & -0.92866770 \\
-200 & 0.07535554 & 0.076248 & -1.17047005 \\
-300 & 0.07353154 & 0.074153 & -0.83807803 \\
-400 & 0.07170754 & 0.071937 & -0.31897355 \\
-500 & 0.06988354 & 0.068829 & 1.53211582 \\
\hline
\end{tabular}


rtigos/Sensores_Teoria_e_Aplicacoes.pdf>. Acesso em: 02 jun. $201 \overline{7}$.

\section{Considerações finais}

Este trabalho objetivava provar que os mesmos fundamentos teóricos usados no medidor sonda lambda para medir nível de oxigênio são capazes achar uma relação direta entre sua tensão de saída e a pressão de ar do ambiente medido, de forma a se obter uma possível função para a resposta do sensor. Já que pelos experimentos, foi possível de se provar essa relação entre pressão e tensão de saída existe, como foi mostrado pela equação 4 , o objetivo inicial foi alcançado.

\section{Referências}

[1] ADAMOWSKI, Julio Cezar et al. Sensores: teoria e aplicações. São Paulo: Redes Cooperativas de Engenharia, [2010]. Disponível em: <http://www.atcp.com.br/imagens/produtos/ceramicas/a
[2] MARKETS AND MARKETS (Org.). Pressure sensor market by technology (Piezoresistive, capacitive, electromagnetic, others), application (Automotive, medical, consumer electronics, others) \& Geography (North America, South America, Europe, APAC, RoW) Global Forecast to 2020. [s. I.]: Markets and Markets, 2015. 166 p. Disponível em: <http://www.researchandmarkets.com/reports/3350635/ pressure-sensor-market-by-technology>. Acesso em: 02 jun. 2017.

[3] COHN, Pedro Estéfano. Analisadores Industriais. Rio de Janeiro: Interciência, 2006.

[4] BROWN, Theodore L. et al. Química: a ciência central. 9. ed. São Paulo: Pearson, 2005.

[5] RIGONATTO, Marcelo. Coeficiente de variação. Disponível em:

<http://mundoeducacao.bol.uol.com.br/matematica/coef icientevariacao.htm>. Acesso em: 31 maio 2017. 